\title{
ON THE PROJECTIVE TENSOR PRODUCT OF FRÉCHET SPACES
}

\author{
by JUAN C. DÍAZ and JUAN A. LÓPEZ MOLINA
}

(Received 13th February 1989)

\begin{abstract}
We are concerned with the following problem. Let $F$ be a Fréchet Montel space and let $E$ be a Fréchet space with a certain property $(P)$. When does it follow that the complete projective tensor product $F \hat{\otimes}$ has the property $(P)$ ? (We consider the following properties: being Montel, reflexive, satisfying the density condition.) In this paper we provide a positive answer if $F$ is a Montel generalized Dubinsky sequence space with decreasing steps.
\end{abstract}

1980 Mathematics subject classification (1985 Revision): 46M05, 46A14.

It is well known that the projective tensor product of two Frechet spaces $E$ and $F$ rarely inherits the good properties of both $E$ and $F$, e.g., $l^{2} \hat{\otimes} l^{2}$ is not reflexive and its projective tensor basis is neither unconditional nor shrinking (cf. [10,13 and 21]). More recently, in [23], Taskinen constructed a Fréchet Montel space $F$ such that $F \hat{\otimes} F$ is not Montel (even more, it is not reflexive). In [25] Taskinen shows that $F$ can be chosen in such a way that $F \hat{\otimes} F$ is not even distinguished. So it seems interesting to find wide classes of Fréchet spaces having a "good" behaviour with respect to the formation of projective tensor products. In this paper, we introduce a class $\Omega$ of Fréchet sequence spaces containing the Köthe echelon spaces $\lambda^{p}(1 \leqq p<\infty$, or $p=0$ and all Montel Dubinsky generalized echelon spaces with decreasing steps. It also contains the sequence spaces considered by Taskinen in his thesis to give positive answers to the problem of topologies of Grothendieck. We show that given a Montel space $\lambda$ in $\Omega$ and a Fréchet space $E$ with a certain property $(P)$, then $\lambda \hat{\otimes} E$ has property $(P)$. We consider the following properties $(P)$ : being Montel, reflexive, satisfying the density condition (cf. [1]), not containing $l^{1}$ (resp. $c_{0}$ ), having a shrinking (resp. boundedly complete) basis. New examples of Fréchet-Montel sequence spaces $\lambda$ in $\Omega$ and Fréchet spaces $E$ such that $L_{b}\left(\lambda, E^{\prime}\right)$ is not $(g D F)$ are also given (see [4]).

In Section 1 we introduce the class $\Omega$ and characterize the Montel spaces in this class. Section 2 is devoted to the technical preliminaries. The main results are derived in Section 3. In the Appendix we include some results which are needed in Section 3.

Our notation is standard. Given Fréchet spaces $E$ and $F$, we denote by $L_{b}\left(E, F^{\prime}\right)$ the space of all linear continuous maps from $E$ into $F^{\prime}$, provided with the topology of uniform convergence on all bounded subsets of $E$. The space $L_{b}(E, \mathbb{R})$, where $\mathbb{R}$ is the field of real numbers, is denoted by $E^{\prime} . E \otimes F$ and $E \otimes F$ denote the projective tensor 
product of $E$ and $F$, and its completion respectively. The topology of a Fréchet space $E$ is always assumed to be generated by an increasing fundamental system of seminorms (f.s.s.), i.e. $\left(p_{k}\right)_{k \in \mathbb{N}}$. with $p_{k} \leqq p_{k+1}(k \in \mathbb{N})$. We denote by $e_{i}$ the canonical $i$ th unit vector in $\omega$. We write $e_{i}^{\prime}$ to mean that $e_{i}$ belongs to the dual of a sequence space. For other unexplained notation in the paper we refer to [14], [15] and [19].

\section{On certain sequence spaces}

We deal with the class $\Omega$ of those Fréchet sequence spaces $\lambda$ for which $\left(e_{i}\right)_{i \in N}$ constitutes an unconditional basis and whose topology is generated by a f.s.s. $\left(p_{k}\right)_{k \in N}$ satisfying the following condition (see [22,3. p. 8]).

$(c \Omega)$ For every $k, t \in \mathbb{N}(k<t)$ there is a constant $M_{k t}>0$ so that, given $A \subset \mathbb{N}$ and given $C \in \mathbb{R}^{+}$such that $p_{k}\left(e_{i}\right) \leqq C p_{t}\left(e_{i}\right)(i \in A)$, then $p_{k}(x) \leqq C M_{k t} p_{t}(x)$ for all $x \in \overline{s p\left\{e_{i} ; i \in A\right\}}$.

We may assume without loss of generality that the seminorms are monotone in the lattice structure induced by the unconditional basis $\left(e_{i}\right)_{i \in N}$ (i.e., given $\left(x_{i}\right)$ and $\left(y_{i}\right) \in \lambda$ with $\left.\left|x_{i}\right| \leqq\left|y_{i}\right|(i \in \mathbb{N}), p_{k}\left(\left(x_{i}\right)\right) \leqq p_{k}\left(\left(y_{i}\right)\right),(k \in \mathbb{N})\right)$. Indeed, if not, we take the seminorms

$$
q_{k}\left(\left(x_{i}\right)\right)=\sup \left\{p_{k}\left(\left(y_{i}\right)\right) ;\left|\left(y_{i}\right)\right| \leqq\left|\left(x_{i}\right)\right|\right\}
$$

(see $[26,1.15])$, and the f.s.s. $\left(q_{k}\right)_{k \in \mathrm{N}}$ also satisfies condition $(c \Omega)$.

Examples of Fréchet spaces in $\Omega$ are Dubinsky's sequence spaces $\lambda=\bigcap_{k \in N}\left(1 / a_{k}\right) \lambda_{k}$, with decreasing stepspaces (i.e. $\lambda_{k+1} \subset \lambda_{k}, k \in \mathbb{N}$ ) such that the coordinate vectors $\left(e_{i}\right)_{i \in \mathbb{N}}$ are an unconditional basis and the $\alpha$-dual $\lambda^{x}$ coincides with $\lambda^{\prime}$ (both conditions are satisfied if $\lambda$ is Montel) (cf. [9], see also [18, p. 166]). In particular, the Köthe echelon spaces $\lambda^{p}, 1 \leqq p<\infty$, or $p=0$, belong to $\Omega$. The class of Fréchet sequence spaces considered by Taskinen in $[22,3]$ is also (strictly) included in $\Omega$.

Given a sequence space $\lambda \in \Omega$ with seminorms $\left(p_{k}\right)_{k \in \mathbb{N}}$ we put $a_{n k}:=p_{k}\left(e_{n}\right), n, k \in \mathbb{N}$. The matrix $\left(a_{n k}\right)_{n, k \in N}$ is said to be strongly increasing if there is no infinite subsequence $J$ of $\mathbb{N}$ and $k_{0}$ such that the sequence $\left(a_{j k_{0}}\right)_{j \in J}$ dominates each sequence $\left(a_{j k}\right)_{j \in J}$ (i.e., there does not exist a sequence $\left(M_{k}\right)_{k}$ of positive numbers such that $a_{j k} \leqq M_{k} a_{j k_{0}}$, for all $j \in J$, $k \in \mathbb{N})$.

The next proposition characterizes the Montel spaces in $\Omega$ and will be needed in the sequel.

Proposition 1.1. Let $\lambda$ be in $\Omega$. The following conditions are equivalent.

(i) $\lambda$ is Montel.

(ii) The matrix $\left(a_{n k}\right)_{n, k \in N}$ is strongly increasing.

Proof. (i) $\Rightarrow$ (ii) If there is a sequence $J \subset \mathbb{N}$ and $k_{0} \in \mathbb{N}$ such that $\left(a_{j k_{0}}\right)_{j \in J}$ dominates every $\left(a_{j k}\right)_{j \in J}$, then the sequence $\left(e_{j} / a_{j k_{0}}\right)_{j \in J}$ is weakly convergent to 0 but does not converge in the topology of $\lambda$, contradicting (i). 
(ii) $\Rightarrow$ (i) We omit the proof of this implication which is very similar to the one of $[15$, I.30.9] (see also $[9,2.5]$ ). It can also be derived from our Lemma 2.1 below.

\section{Technical preparations}

We first introduce further notation and some remarks. Given a sequence $\left(x_{i}\right)_{i \in N} \in \omega$. and given $D \subset \mathbb{N}, S_{D}\left(x_{i}\right)$ stands for the sequence $\left(\bar{x}_{i}\right)_{i \in \mathbb{N}}$ defined by $\bar{x}_{i}:=x_{i}$ if $i \in D$, and $\bar{x}_{i}:=0$ otherwise. Given $\lambda \in \Omega$ and $D \subset \mathbb{N}$, then $S_{D}$ defines a continuous linear projection from $\lambda$ onto the sectional subspace $\lambda_{D}=\left\{S_{D}(x) ; x \in \lambda\right\}$.

Given $\lambda \in \Omega$ and a Fréchet space $E$, then $e_{i}^{\prime}$ will be identified with a continuous linear mapping from $\lambda \hat{\otimes} E$ into $E$ in the following way:

$$
e_{i}^{\prime}\left(\sum_{h=1}^{\infty} x_{h} \otimes w_{h}\right):=\sum_{h=1}^{\infty} e_{i}^{\prime}\left(x_{h}\right) w_{h}\left(\equiv \sum_{h=1}^{\infty} x_{i h} w_{h}\right), \quad \text { where } \quad x_{h}=\left(x_{j h}\right)=\sum_{j=1}^{\infty} x_{j h} e_{j}, \quad(h \in \mathbb{N})
$$

We also recall that any element $f \in(\lambda \hat{\otimes} E)^{\prime}$ can be considered as a continuous linear mapping from $\lambda$ into $E^{\prime}$, as follows: given $x \in \lambda$, we define $f(x): E \rightarrow \mathbb{R}$ by $y \mapsto\langle x \otimes y, f\rangle$. With these identifications, given $z=\sum_{h=1}^{\infty}\left(x_{j h}\right) \otimes w_{h} \in \lambda \hat{\otimes} E, f \in(\lambda \hat{\otimes} E)^{\prime}$, and $i \in \mathbb{N}$, we can write $\left\langle e_{i}^{\prime}(z), f\left(e_{i}\right)\right\rangle$ as meaning $\sum_{h=1}^{\infty} x_{i h}\left\langle w_{h}, f\left(e_{i}\right)\right\rangle=\sum_{h=1}^{\infty} x_{i h}\left\langle e_{i} \otimes w_{h}, f\right\rangle$.

The next result is the key to the remainder of the paper.

Lemma 2.1. Let $\lambda$ be a sequence space in $\Omega$, with $\left(a_{n k}\right)_{n, k \in N}$ strongly increasing, and let $E$ be a Fréchet space. Let $\left(z_{\alpha}\right)_{\alpha \in A}$ and $\left(f_{\alpha}\right)_{\alpha \in A}$ be bounded nets in $\lambda \hat{\otimes} E$ and $(\lambda \hat{\otimes} E)^{\prime}$ respectively, so that

$$
\lim _{\alpha}\left\langle e_{i}^{\prime}\left(z_{\alpha}\right), f_{\alpha}\left(e_{i}\right)\right\rangle=0, \quad \forall i \in \mathbb{N}
$$

Then

$$
\lim _{\alpha}\left\langle z_{\alpha}, f_{\alpha}\right\rangle=0
$$

Proof. If the assertion were not true, there would be $\varepsilon>0$ and a subnet of $A$ (denoted by $A$ again) such that $\left|\left\langle z_{\alpha}, f_{\alpha}\right\rangle\right|>\varepsilon(\alpha \in A)$. Let us choose representations $z_{\alpha}=\sum_{j=1}^{\infty}\left(x_{i j}^{\alpha}\right) \otimes w_{j}^{\alpha}(\alpha \in A)$. Then, we can write our hypothesis and the inequality above as follows:

$$
\lim _{\alpha}\left\langle e_{i}^{\prime}\left(z_{\alpha}\right), f_{\alpha}\left(e_{i}\right)\right\rangle=\lim _{\alpha} \sum_{j=1}^{\infty} x_{i j}^{\alpha}\left\langle w_{j}^{\alpha}, f_{\alpha}\left(e_{i}\right)\right\rangle=0, \quad \forall i \in \mathbb{N}
$$

and

$$
\left|\left\langle z_{\alpha}, f_{\alpha}\right\rangle\right|=\left|\sum_{j=1}^{\infty}\left(\sum_{i=1}^{\infty} x_{i j}^{\alpha}\left\langle w_{j}^{\alpha}, f_{\alpha}\left(e_{i}\right)\right\rangle\right)\right|>\varepsilon, \quad \forall \alpha \in A
$$


From this inequality, for every $\alpha \in A$ and $n \in \mathbb{N}$ there are $h_{\alpha}, m_{\alpha}>n$ such that

$$
\left|\sum_{j=1}^{h \alpha} \sum_{i=1}^{m \alpha} x_{i j}^{\alpha}\left\langle w_{j}^{\alpha}, f_{\alpha}\left(e_{i}\right)\right\rangle\right|>\varepsilon, \quad \forall \alpha \in A .
$$

From now on we fix f.s.s. $\left(p_{k}\right)_{k \in N}$ and $\left(q_{k}\right)_{k \in N}$ in $\lambda$ and $E$ respectively. We divide the proof into three steps.

1. There are sequences $(\alpha(r))_{r \in \mathbb{N}}$ in $A$, and $(h(r))_{r \in \mathbb{N}},(m(r))_{r \in \mathbb{N}}$ in $\mathbb{N}$, with $(m(r))_{r \in N}$ strictly increasing, such that for every $r \in \mathbb{N}$ one has

$$
\left|\sum_{j=1}^{h(r)} \sum_{i=m(r)+1}^{m(r+1)} x_{i j}^{\alpha(r)}\left\langle w_{j}^{\alpha(r)}, f_{\alpha(r)}\left(e_{i}\right)\right\rangle\right|>\varepsilon
$$

and

$$
\left(p_{r} \otimes q_{r}\right)\left(z_{\alpha(r)}-\sum_{j=1}^{h(r)}\left(x_{i j}^{\alpha(r)}\right) \otimes w_{j}^{\alpha(r)}\right) \leqq 1
$$

Indeed, the result follows by (1) and (2) and by using a "sliding hump" argument. The details are left to the reader.

2. We put $\bar{z}_{r}:=\sum_{j=1}^{h(r)}\left(x_{i j}^{\alpha(r)}\right) \otimes w_{j}^{\alpha(r)}$. By $\left(3^{\prime}\right)$, the sequence $\left(\bar{z}_{r}\right)_{r \in \mathrm{N}}$ is bounded, whence, for every $k \in \mathbb{N}$, we can choose representations $\bar{z}_{r}=\sum_{j}\left(x_{i j}^{r k}\right) \otimes w_{j}^{r k}$ such that $\sum_{j} p_{k}\left(\left(x_{i j}^{r k}\right)\right) q_{k}\left(w_{j}^{r k}\right)<T_{k}$, for some $T_{k} \in \mathbb{R}^{+}$not depending on $r$. The net $\left(f_{\alpha}\right)_{\alpha \in A}$ is bounded, so there is $k_{0} \in \mathbb{N}$ such that $\left|\left\langle z, f_{\alpha}\right\rangle\right| \leqq\left(p_{k_{0}} \otimes q_{k_{0}}\right)(z)$, for all $z \in \lambda \otimes E$. We then take scalars $d_{k} \in \mathbb{R}^{+}, k \geqq k_{0}$, such that $\sum_{k=k_{0}}^{\infty} M_{k_{0} k} / d_{k}=1 / 2$, where $M_{k_{0} k}$ are the constants in $(c \Omega)$, and, for every $k, s \in \mathbb{N}$, we set

$$
T_{k s}:=\left\{i \in \mathbb{N} ; m(s)+1 \leqq i \leqq m(s+1) \quad \text { and } \quad a_{i k}>\left(T_{k} d_{k} / \varepsilon\right) a_{i k_{0}}\right\}
$$

We now assert that, given $k \geqq k_{0}, s \in \mathbb{N}$, and $D \subset T_{k s}$, we get

$$
\left(p_{k_{0}} \otimes q_{k_{0}}\right)\left(S_{D} \otimes 1_{E}\left(\bar{z}_{r}\right)\right)<\varepsilon M_{k_{0} k} / d_{k}, \quad \forall r \in \mathbb{N} .
$$

In fact, by the monotony of the seminorms $\left(p_{k}\right)_{\in N}$ and by $(c \Omega)$, we have

$$
\begin{aligned}
T_{k}>\sum_{j} p_{k}\left(\left(x_{i j}^{r k}\right)\right) q_{k}\left(w_{j}^{r k}\right) & \geqq \sum_{j} p_{k}\left(S_{D}\left(x_{i j}^{r k}\right)\right) q_{k}\left(w_{j}^{r k}\right) \\
& \geqq\left(M_{k_{0} k}^{-1} T_{k} d_{k} / \varepsilon\right) \sum_{j} p_{k_{0}}\left(S_{D}\left(x_{i j}^{r k}\right)\right) q_{k_{0}}\left(w_{j}^{r k}\right) \\
& \geqq\left(M_{k_{0} k}^{-1} T_{k} d_{k} / \varepsilon\right)\left(p_{k_{0}} \otimes q_{k_{0}}\right)\left(S_{D} \otimes 1_{E}\left(\bar{z}_{r}\right)\right)
\end{aligned}
$$


3. For each $s \in \mathbb{N}$, there is a natural number $i_{s}$, with $m(s)+1 \leqq i_{s} \leqq m(s+1)$ and such that $i_{s} \notin T_{k s}$, for all $k \geqq k_{0}$.

If not, there would be $k_{1}, \ldots, k_{t} \geqq k_{0}$ such that $\{i ; m(s)+1 \leqq i \leqq m(s+1)\}=\bigcup_{i=1}^{t} T_{k i s}=$ $\bigcup_{i=1}^{t} D_{k_{i} s}$, where the sets $D_{k_{i} s}$ are pairwise disjoint and $D_{k_{i} s} \subset T_{k_{i} s}, 1 \leqq i \leqq t$. Then, by (3) and (4) one would have

$$
\begin{aligned}
\varepsilon / 2 & <\left|\sum_{j=1}^{h(s)} \sum_{i=m(s)+1}^{m(s+1)} x_{i j}^{\alpha(s)}\left\langle w_{j}^{\alpha(s)}, f_{\alpha(s)}\left(e_{i}\right)\right\rangle\right| \\
& =\left|\sum_{j=1}^{h(s)} \sum_{n=1}^{t} \sum_{i \in D_{k_{n} s}} x_{i j}^{\alpha(s)}\left\langle w_{j}^{\alpha(s)}, f_{\alpha(s)} c\left(e_{i}\right)\right\rangle\right| \\
& \leqq \sum_{n=1}^{t}\left|\left\langle\sum_{j=1}^{h(s)} S_{D_{k_{s, s}}}\left(x_{i j}^{\alpha(s)}\right) \otimes w_{j}^{\alpha(s)}, f_{\alpha(s)}\right\rangle\right| \\
& \leqq \sum_{n=1}^{t}\left(p_{k_{0}} \otimes q_{k_{0}}\right)\left(S_{D_{k_{n} s}} \otimes 1_{E}\left(\bar{z}_{s}\right)\right) \leqq \sum_{n=1}^{t} \varepsilon M_{k_{0} k_{n}} / d_{k_{n}}<\varepsilon / 2 .
\end{aligned}
$$

This contradiction establishes our assertion 3. Hence we can take a subsequence $\left(i_{s}\right)_{s \in \mathrm{N}}$ such that $\left(a_{i_{s} k_{0}}\right)_{s \in \mathbb{N}}$ dominates each $\left(a_{i_{s} k}\right)_{s \in N}$ contradicting the fact that $\left(a_{n k}\right)_{n, k \in N}$ is strongly increasing.

As a first consequence we obtain the following proposition from which we shall derive most of the main results.

Proposition 2.2. Let $\lambda$ be a Montel space in $\Omega$, let $E$ be a Fréchet space and let $\left(z_{\alpha}\right)_{a \in A}$ and $\left(f_{\alpha}\right)_{\alpha \in A}$ be bounded nets in $\lambda \hat{\otimes} E$ and $(\lambda \hat{\otimes} E)^{\prime}$ respectively. Then:

(a) $\left(z_{\alpha}\right)_{\alpha \in A}\left(\right.$ resp. $\left.\left(f_{\alpha}\right)_{\alpha \in A}\right)$ converges to 0 whenever $\left(e_{i}^{\prime}\left(z_{\alpha}\right)\right)_{\alpha \in A}\left(\right.$ resp. $\left.\left(f_{\alpha}\left(e_{i}\right)\right)_{\alpha \in A}\right)$ converges to 0 for every $i \in \mathbb{N}$;

(b) $\left(z_{\alpha}\right)_{\alpha \in A}$ converges weakly to 0 (resp. $\left(f_{\alpha}\right)_{\alpha \in A}$ is weak ${ }^{*}$-convergent to 0$)$ if $\left(e_{i}^{\prime}\left(z_{\alpha}\right)\right)_{\alpha \in A}$ converges weakly to $0\left(\right.$ resp. $\left(f_{\alpha}\left(e_{i}\right)\right)_{\alpha \in A}$ is weak ${ }^{*}$-convergent to 0$)$, for all $i \in \mathbb{N}$;

(c) $\left(z_{\alpha}\right)_{\alpha \in A}\left(\right.$ resp. $\left.\left(f_{a}\right)_{\alpha \in A}\right)$ is Cauchy whenever $\left(e_{i}^{\prime}\left(z_{a}\right)\right)_{\alpha \in A}\left(\right.$ resp. $\left.\left(f_{\alpha}\left(e_{i}\right)\right)_{\alpha \in A}\right)$ is Cauchy, for all $i \in \mathbb{N}$. The same holds in the weak (resp. weak*) topology.

Proof. (a) Take $\left(z_{\alpha}\right)_{\alpha \in A}$ bounded in $\lambda \hat{\otimes} E$ and so that $\left(e_{i}^{\prime}\left(z_{\alpha}\right)\right)_{\alpha \in A}$ converges to 0 in $E$ $(i \in \mathbb{N})$. Let us see that $\left(z_{\alpha}\right)_{\alpha \in A}$ converges to 0 . To this end we take $\left(f_{\alpha}\right)_{a \in A}$ bounded in $(\lambda \hat{\otimes} E)^{\prime}$ and we show that $\left(\left\langle z_{\alpha}, f_{\alpha}\right\rangle\right)_{\alpha \in A}$ converges to 0 . Indeed, for every $i \in \mathbb{N},\left(f_{\alpha}\left(e_{i}\right)\right)_{\alpha \in A}$ is bounded in $E^{\prime}$, whence 


$$
\lim _{\alpha \in A}\left(\sup _{\delta \in A}\left|\left\langle e_{i}^{\prime}\left(z_{\alpha}\right), f_{\delta}\left(e_{i}\right)\right\rangle\right|\right)=0, \quad \forall i \in N
$$

Thus, in particular, $\lim _{\alpha}\left\langle e_{i}^{\prime}\left(z_{\alpha}\right), f_{\alpha}\left(e_{i}\right)\right\rangle=0,(i \in \mathbb{N})$. We now apply Lemma 2.1 .

The proof of the dual case is analogous.

(b) Assume now that $\left(e_{i}^{\prime}\left(z_{\alpha}\right)\right)_{\alpha \in A}$ is $\sigma\left(E, E^{\prime}\right)$-convergent to 0 , for every $i \in \mathbb{N}$. Take any $f \in(\lambda \otimes E)^{\prime}$, and set $f_{\alpha}:=f,(\alpha \in A)$. Then

$$
\lim _{\alpha}\left\langle e_{i}^{\prime}\left(z_{\alpha}\right), f_{\alpha}\left(e_{i}\right)\right\rangle=\lim _{\alpha}\left\langle e_{i}^{\prime}\left(z_{\alpha}\right), f\left(e_{i}\right)\right\rangle=0, \quad \forall i \in \mathbb{N}
$$

thus, $\lim _{\alpha}\left\langle z_{\alpha}, f\right\rangle=0$ by applying Lemma 2.1 , and the assertion follows.

The dual case is similar.

(c) is readily checked using (a) and (b).

\section{Main results}

Taskinen ([23]) gave an example of a Fréchet Montel space $F$ such that $F \hat{\otimes} F$ is not Montel (even more, it is not reflexive). On the other hand, there are examples of pairs of Fréchet spaces $E$ and $F$ such that $E \hat{\otimes} F$ is Montel (see [3, Corollary (1)], [18] and [23, 3.3]). Our theorem below extends these results, and it also improves [3, Corollary (2)] and $[18$, Theorem $4,(2)]$.

Theorem 3.1. Let $\lambda$ be a Montel space in $\Omega$ and let $E$ be a Fréchet space with a certain property $(P)$. Then $\lambda \hat{\otimes} E$ has the property $(P)$ whenever $(P)$ is:

(i) the Montel property,

(ii) non-containment of $l^{1}$ (resp. $\left.c_{0}\right)$,

(iii) reflexivity,

(iv) having a shrinking (resp. boundedly complete) basis.

Proof. (i) $\lambda \hat{\otimes} E$ is separable so we must only show that strongly convergent and weak $^{*}$-convergent sequences coincide in $(\lambda \hat{\otimes} E)^{\prime}$. To do this, take a weak*-null sequence $\left(f_{n}\right)_{n \in \mathbb{N}}$ in $(\lambda \widehat{\otimes} E)^{\prime}$. One readily checks that $\left(f_{n}\left(e_{i}\right)\right)_{n \in N}(i \in \mathbb{N})$ is weak*-null in $E^{\prime}$, hence it is strongly convergent to 0 since $E$ is Montel. We now apply Proposition 2.2(a).

(ii) First assume that $E$ contains no copy of $l^{1}$ and let $\left(z_{n}\right)_{n \in N}$ be a bounded sequence in $\lambda \hat{\otimes} E$. We are done if we show that $\left(z_{n}\right)_{n \in N}$ has a weak Cauchy subsequence (see Proposition A.1 in our Appendix). Indeed, $\left(e_{i}^{\prime}\left(z_{n}\right)\right)_{n \in N}$ is bounded in $E$, whence it has a weak Cauchy subsequence, for all $i \in \mathbb{N}$. Then, by using induction and a diagonal procedure, we find a subsequence of $\left(z_{n}\right)_{n \in N}$, say $\left(z_{n j}\right)_{j \in N}$, such that $\left(e_{i}^{\prime}\left(z_{n j}\right)\right)_{j \in N}$ is weak Cauchy for every $i \in \mathbb{N}$. Thus $\left(z_{n_{j}}\right)_{j \in \mathbb{N}}$ is weak Cauchy by Proposition 2.2(c).

The proof of the $c_{0}$-case can be checked by the reader in a similar way by using Proposition A.2. 
(iii) According to (ii), no subspace of $\lambda \hat{\otimes} E$ is isomorphic either to $c_{0}$ or to $l^{1}$. Thus, $\lambda \otimes E$ is weakly sequentially complete by Lemma A.4 and Theorem A.5, whence it is reflexive by Proposition A.1.

(iv) Let $\left(x_{n}\right)_{n \in N}$ be a basis (by a basis we always mean a Schauder basis here) in $E$. Let us recall that $\left(e_{i} \otimes x_{j}\right)_{\Phi(i, j)=1}^{\infty}$ is a Schauder basis of $\lambda \hat{\otimes} E$, where $\Phi(i, j)$ denotes the upper rectangular ordering of $\mathbb{N} \times \mathbb{N}$ (i.e., $\Phi(1,1)=1, \Phi(1,2)=2, \Phi(2,2)=3, \ldots$ ). As a consequence, $\left(e_{i}^{\prime} \otimes x_{j}^{\prime}\right)_{\Phi(i, j)=1}^{\infty}$ is a basis of $(\lambda \widehat{\otimes} E)^{\prime}$ in the weak*-topology, where $\left(x_{j}^{\prime}\right)_{j \in \mathbb{N}}$ are the associated coefficient functionals. Now let us assume that $\left(x_{n}\right)_{n \in \mathbb{N}}$ is shrinking (see $[14,14]$ for the definitions). Take any $f \in(\lambda \hat{\otimes} E)^{\prime}$ and let $\left(c_{i j}\right)_{i, j \in N}$ be the matrix associated with $f$ (i.e., $c_{i j}:=f\left(e_{i} \otimes x_{j}\right), i, j \in \mathbb{N}$ ). We set $f_{n}:=\sum_{\Phi(i, j)=1}^{n} c_{i j} e_{i}^{\prime} \otimes x_{j}^{\prime}$. The sequence $\left(f-f_{n}\right)_{n \in N}$ is weak*-null, hence bounded. Furthermore, since $\left(x_{j}^{\prime}\right)_{j \in N}$ is a basis in $E^{\prime}$, it follows that $\left(f-f_{n}\left(e_{i}\right)\right)_{n \in \mathbb{N}}$ is strongly convergent to $0(i \in \mathbb{N})$. Thus, $\left(f-f_{n}\right)_{n \in \mathbb{N}}$ is strongly convergent to 0 by Proposition $2.2(\mathrm{a})$. Consequently $\left(e_{i}^{\prime} \otimes x_{j}^{\prime}\right)_{(i, j)=1}^{\infty}$ is a basis in $(\lambda \hat{\otimes} E)^{\prime}$.

Let $\left(x_{n}\right)_{n \in N}$ now be a boundedly complete basis in $E$. Take a bounded sequence $z_{n}=\sum_{\mathbb{\Phi}(i, j)=1}^{n} \alpha_{i j} e_{i} \otimes x_{j}, n \in \mathbb{N}$, and let us see that it is Cauchy. Indeed, for any $i \in \mathbb{N}$, we set $F_{i m}:=\{j ; \Phi(i, j) \leqq m\}$. Then, $\left(e_{i}^{\prime}\left(z_{n}\right)\right)_{n \in N}\left(\equiv\left(\sum_{j \in F_{i n}} \alpha_{i j} x_{j}\right)_{n \in N}\right)$ is bounded in $E$, hence $\sum_{j=1}^{\infty} \alpha_{i j} x_{j}$ converges in $E$, so $\left(e_{i}^{\prime}\left(z_{n}\right)\right)_{n \in N}$ converges. The index $i$ is arbitrary, whence $\left(z_{n}\right)_{n \in N}$ is Cauchy by Proposition 2.2(c).

We finish this section with a further result related to the problem of topologies of Grothendieck. Given two Fréchet spaces $E$ and $F$, the projective tensor product $E \hat{\otimes} F$ is said to have the property $(B B)$ (cf. [22]) whenever every bounded set $B$ of $E \hat{\otimes} F$ is contained in a set $\overline{\Gamma(K \otimes L)}$ for some bounded sets $K$ in $E$ and $L$ in $F$. The problem of whether $E \hat{\otimes} F$ has property $(B B)$ for arbitrary Frechet spaces is called the problem of topologies of Grothendieck and it was recently solved, in the negative, by Taskinen (see $[22,23,24])$. We recall that $(E \hat{\otimes} F)^{\prime}$ and $L_{b}\left(E, F^{\prime}\right)$ can be (algebraically) identified in a canonical way as follows: $I: L_{b}\left(E, F^{\prime}\right) \rightarrow(E \hat{\otimes} F)^{\prime}, \quad\left\langle\sum_{i} x_{i} \otimes y_{i}, I(f)\right\rangle:=\sum_{i}\left\langle y_{i}, f\left(x_{i}\right)\right\rangle$, $\left(f \in L_{b}\left(E, F^{\prime}\right)\right) . I$ is open and it makes the families of bounded sets correspond to each other. Moreover, $I$ is continuous if and only if $E \widehat{\otimes} \mathrm{F}$ has property $(B B)($ see $[8,3.4])$. Our next result should be compared with $[8,3.5]$ and $[3$, Lemma 3$]$.

Proposition 3.2 Let $\lambda$ be a Montel space in $\Omega$ and let $E$ be a Fréchet space. The canonical mapping $I: L_{b}\left(\lambda, E^{\prime}\right) \rightarrow(\lambda \hat{\otimes} E)^{\prime}$ is an isomorphism when restricted to the bounded sets. In particular, $\lambda \hat{\otimes} E$ has property $(B B)$ whenever $L_{b}\left(\lambda, E^{\prime}\right)$ is a $g D F$-space.

Proof. We must only show that $I$ is continuous when restricted to the bounded sets. Thus, take a bounded net $\left(f_{\alpha}\right)_{\alpha \in A}$ in $L_{b}\left(\lambda, E^{\prime}\right)$, convergent to 0 . Then $\left(I\left(f_{\alpha}\right)\right)_{\alpha \in A}$ is bounded in $(\lambda \hat{\otimes} E)^{\prime}$. Moreover, one can easily check that $\left(I\left(f_{\alpha}\right)\left(e_{i}\right)\right)_{\alpha \in A}\left(\equiv\left(f_{\alpha}\left(e_{i}\right)\right)_{\alpha \in A}\right)$ converges strongly to 0 in $E^{\prime}$, for all $i \in \mathbb{N}$. The conclusion follows from Proposition 2.2(a).

Remarks. (a) A Fréchet space $E$ is said to be an $F B B$-space if $E \hat{\otimes} F$ has property $(B B)$ for each Fréchet space $F$. By the work of Taskinen (cf. [24]), we know that an $F B B$-space must be a $\pi$-space (see [12] for the definition). We also know that a non- 
nuclear, Köthe echelon space $\lambda^{p}$, with $1<p \leqq \infty$ or $p=0$, is not a $\pi$-space (see $[12,1.5]$ and $[20,29.7 .6])$. Consequently, by using Proposition 3.2, we get new counterexamples to an old open problem which was solved by Taskinen resp. Bonet-Galbis (compare with the examples in [4]). Namely, we have: Let $\lambda^{p}(1<p \leqq \infty)$ be a Montel, non-nuclear Köthe echelon space. Then, there is a Fréchet space $E$ such that $L_{b}\left(\lambda^{p}, E^{\prime}\right)$ is not a $(g D F)$-space. By $[22,3.1]$ and by our Theorem 3.1 , the Fréchet space $E$ in the statement above can be neither normable nor Montel. Furthermore, the space $E$ that we know is the one constructed by Taskinen in $[24,3.2 .4]$, which is non-distinguished according to the results of Bonet and Dierolf (cf. [5]), but can even be taken to be reflexive by reformulating the construction (see the forthcoming article by Defant, Floret and Taskinen).

(b) Let us recall that a Fréchet space $E$ has the density condition if and only if the bounded sets in $E^{\prime}$ are metrizable (cf. [1]). It follows from $[2,6]$ that $L_{b}\left(E, F^{\prime}\right)$ has a fundamental sequence of bounded sets which are metrizable whenever $E$ and $F$ are Fréchet spaces with the density condition. From this and the first part of Proposition 3.2, we get: Let $\lambda$ be a Montel space in $\Omega$ and let $E$ be a Fréchet space satisfying the density condition. Then $\lambda \hat{\otimes} E$ satisfies the density condition.

\section{Appendix}

The following propositions generalize, from Banach to Fréchet spaces, well known results. The first one was proved in [7]. The second one is an extension of $[16,2 . e .4]$ and can be readily checked by the reader.

Proposition A.1. A bounded sequence in a Fréchet space either has a weak-Cauchy subsequence or a subsequence which is a basic sequence equivalent to the unit vector basis of $l^{1}$.

As a consequence, $c$ : weakly sequentially complete Fréchet space is reflexive if and only if it contains no copy of $l^{1}$.

Proposition A.2. A Fréchet space E contains a copy of $c_{0}$ if and only if there is a sequence $\left(z_{n}\right)_{n \in N}$ in $E$ such that $\sum_{n=1}^{\infty}\left|\left\langle z_{n}, x^{\prime}\right\rangle\right|<\infty$ for every $x^{\prime} \in E^{\prime}$, the set $\left\{\sum_{j=1}^{n} z_{j} ; n \in \mathbb{N}\right\}$ is bounded and $\sum_{n=1}^{\infty} z_{n}$ diverges.

An (unconditional) Schauder decomposition of a Fréchet space $E$ is a sequence of continuous projections $\left(Q_{k}\right)_{k \in N}, Q_{k}: E \rightarrow E$, such that $Q_{j} Q_{k}=\delta_{j k} Q_{k}$ and $\sum_{k=1}^{\infty} Q_{k}(z)=z$ for all $z \in E$, where the series converges unconditionally. The decomposition is said to be boundedly complete if a series $\sum_{k=1}^{\infty} z_{k}$ converges whenever $z_{k} \in Q_{k}(E)(k \in \mathbb{N})$ and $\left\{\sum_{j=1}^{n} z_{j} ; n \in \mathbb{N}\right\}$ is bounded.

Remark. If $\left(Q_{n}\right)_{n \in N}$ is an unconditional decomposition in a Fréchet space $E$, and we denote by $\Delta$ the set of all scalar sequences $\delta$ such that $\left|\delta_{n}\right|=1(n \in \mathbb{N})$ then the family of operators $\left\{\sum_{j \in M} \delta_{j} Q_{j} ; \delta \in \Delta, M \subset \mathbb{N}\right\}$ is equicontinuous (the proof is similar to that of $[14,14.7 .31]$; also see $[26])$.

The next two lemmata are easy extensions of [11], Lemma 1 and Lemma 2 respectively: 
Lemma A.3. Let $E$ be a Fréchet space and $\left(Q_{k}\right)_{k \in N}$ an unconditional decomposition on $E$. $A$ weak Cauchy sequence $\left(z_{n}\right)_{n \in N}$ in $E$ is weakly convergent to $z_{0} \in E$ if and only if $\left(Q_{k}\left(z_{n}\right)\right)_{n \in \mathbb{N}}$ converges weakly to $Q_{k}\left(z_{0}\right)$ for every $k \in \mathbb{N}$.

Lemma A.4. Let $E$ and $F$ be Fréchet spaces such that $E$ has an unconditional basis $\left(x_{n}\right)_{n \in N}$. Then, the sequence $\left(Q_{k}\right)_{k \in N}$ of canonical projections, $Q_{k}: E \hat{\otimes} F \rightarrow\left\{x_{k}\right\} \otimes F$, is an unconditional decomposition of $E \hat{\otimes} F$.

Theorem A.5. Let $E$ be a Fréchet space with an unconditional decomposition $\left(Q_{k}\right)_{k \in N}$ such that $Q_{k}(E)$ is weakly sequentially complete $(k \in \mathbb{N})$. Then, the following are equivalent:

(a) the decomposition is boundedly complete,

(b) $E$ is weakly sequentially complete,

(c) E contains no copy of $c_{0}$.

Proof. (a) $\Rightarrow$ (b) Let $\left(y_{n}\right)_{n \in N}$ be a weak Cauchy sequence in $E$. Then, for every $k \in \mathbb{N}$, $\left(Q_{k}\left(y_{n}\right)\right)_{n \in \mathcal{N}}$ is weak Cauchy in $Q_{k}(E)$, so weakly convergent to some $z_{k} \in Q_{k}(E)$. Now, $\left\{\sum_{j=1}^{n} z_{j} ; n \in \mathbb{N}\right\}$ is bounded (see the Remark); hence the series $\sum_{n=1}^{\infty} z_{n}$ converges. By Lemma A. 3 it follows that $\left(y_{n}\right)_{n \in N}$ converges to $\sum_{n=1}^{\infty} z_{n}$.

(b) $\Rightarrow$ (c) is known. So it remains to show that (c) implies (a). Indeed, assume that there are elements $z_{n} \in Q_{n}(E)(n \in \mathbb{N})$ so that $\left\{\sum_{j=1}^{n} z_{j} ; j \in \mathbb{N}\right\}$ is bounded but $\sum_{j=1}^{\infty} z_{j}$ diverges. Given any $x^{\prime} \in E^{\prime}$, we take a sequence of scalars $\left(\delta_{n}\right)_{n}$ such that $\left|\left\langle z_{k}, x^{\prime}\right\rangle\right|=\delta_{k}\left\langle z_{k}, x^{\prime}\right\rangle$ $(k \in \mathbb{N})$. The set $\left\{\sum_{j=1}^{n} \delta_{j} z_{j} ; n \in \mathbb{N}\right\}$ is bounded, whence $\sum_{j=1}^{\infty}\left|\left\langle z_{j}, x^{\prime}\right\rangle\right|=\sum_{j=1}^{\infty} \delta_{j}\left\langle z_{j}, x^{\prime}\right\rangle<$ $\infty$. Since $x^{\prime}$ is arbitrary we apply Proposition A.2 to get a subspace of $E$ isomorphic to $c_{0}$, contradicting (c).

Note. M. A. Miñarro and the first-named author have recently proved that Theorem 3.1 remains true if the property $(P)$ is that of being distinguished (see Doga, Tr. J. Math. 14 (1990), 191-208).

Acknowledgements. We thank Prof. Bonet who read the manuscript and made helpful comments. The second-named author was partially supported by the DGCYT Proyecto no. PS88-0050.

\section{REFERENCES}

1. K. D. Bierstedt and J. Bonet, Stefan Heinrich's density condition for Fréchet spaces and the characterization of the distinguished echelon spaces, Math. Nachr. 135 (1988), 149-180.

2. K. D. Bierstedt and J. Bonet, Density Conditions in Fréchet and (DF)-spaces (Functional Analysis Congress at El Escorial, 1988).

3. J. Bonet and A. Defant, Projective tensor products of distinguished Fréchet spaces, Proc. Roy. Irish Acad., Sect. A 85 (1985), 193-199.

4. J. Bonet and A. Galbis, A note on Taskinen's counterexamples on the problem of topologies of Grothendieck, Proc. Edinburgh Math. Soc. 32 (1989), 281-283.

5. J. Bonet and S. Dierolf, Fréchet Spaces of Moscatelli Type (Functional Analysis Congress at El Escorial, 1988).

6. J. C. Diaz, An example of Fréchet space, not Montel, without infinite dimensional normable subspaces, Proc. Amer. Math. Soc. 96 (1986), 721. 
7. J. C. Diaz, Montel subspaces in countable projective limits of $L^{p}(\mu)$-spaces, Canad. Math. Bull. 32 (1989), 169-176.

8. S. Dierolf, On spaces of continuous linear mappings between locally convex spaces, Note Mat. 5 (1985), 147-255.

9. E. Dubinsky, Perfect Fréchet spaces, Math. Ann. 174 (1967), 186-194.

10. B. R. Gelbaum, J. Gil de Lamadrid, Bases of tensor products of Banach spaces, Pacific $J$. Math. 11 (1961), 1281-1286.

11. S. HeINRICH, Weak sequential completeness of Banach operator ideals, Siberian Math. J. 17 (1976), 857-862.

12. R. Hollstein, Extension and lifting of continuous linear mappings in locally convex spaces, Math. Nachr. 108 (1982), 275-297.

13. J. R. Holub, Tensor product bases and tensor diagonals, Trans. Amer. Math. Soc. 151 (1970), 563-579.

14. H. JARChow, Locally Convex Spaces (Teubner, Stuttgart, 1981).

15. G. KörHe, Topological Vector Spaces I, II (Springer-Verlag, Berlin-Heidelberg-New York, $1969,1979)$.

16. J. Lindenstrauss and L. Tzafirı, Classical Banach Spaces, I (Springer-Verlag, Berlin, 1977).

17. J. A. López-Molina, Reflexivity of projective tensor products of echelon and coechelon Köthe spaces, Collect. Math. 33 (1982), 259-284.

18. J. A. López-Molina, El producto tensorial (p-r-s) proyectivo de espacios perfectos de Fréchet-Montel, Rev. Real Acad. Cience. Madrid 79 (1985), 153-169.

19. P. Perez Carreras and J. Bonet, Barrelled Locally Convex Spaces (North-Holland, Amsterdam, 1987).

20. A. Pietsch, Operator Ideals (North-Holland, Amsterdam, 1980).

21. R. Ryan, The Dunford-Pettis property and projective tensor products, Bull. Acad. Polon. Sci. 27 (1979), 373-379.

22. J. Taskinen, Counterexamples to "problème des topologies" of Grothendieck, Ann. Acad. Sci. Fenn. Ser. A, 63 (1986).

23. J. TASKinen, The projective tensor product of Fréchet Montel spaces, Studia Math. 91 (1988), 17-30.

24. J. TAZSkinen, (FBa)- and (FBB)-spaces, Math. Z. 198 (1988), 339-365.

25. J. Taskinen, Examples of non-distinguished Fréchet spaces, Ann. Acad. Sci. Fenn., to appears.

26. L. J. WeILL, Unconditional and shrinking bases in locally convex spaces, Pacific J. Math. 29 (1969), 467-483.

Cátedra de Matemáticas

E.T.S.I. AGrónOMOS

Universidad de Córdoba

14004 Cordoba, Spain
Ampliación de Matemáticas

E.T.S.I. Agronomos

Universidad Pol. de Valencia 46071 Valencia, Spain 